\title{
Perceptions of Teledermatology among People with Hidradenitis Suppurativa
}

\author{
Ashley Eichelberger, CRNP ${ }^{1}$, Melissa Butt, MPH${ }^{1}$, Colleen Silva, MD ${ }^{1}$, Aretha Mosley ${ }^{1}$, Joslyn \\ S. Kirby, MD, MS, MEd ${ }^{1}$ \\ ${ }^{1}$ Department of Dermatology, Penn State Milton S Hershey Medical Center, Hershey, PA
}

\section{ABSTRACT}

Introduction: Hidradenitis suppurativa (HS) is a chronic inflammatory condition that requires frequent dermatology visits. Coronavirus disease (COVID-19) led to a shift in healthcare delivery to telemedicine. Teledermatology can be valuable for HS patients to decrease travel and wait times and improve patient access. However, we hypothesized that people with HS may have concerns or challenges participating in HS due to its frequent occurrence in sensitive or difficult-to-photograph locations.

Methods: A cross-sectional survey was sent to patients with $\mathrm{HS}$ at one academic institution as well as leaders of three patient networks.

Results: Survey responses were received from 149 patients. A total of $94.4 \%$ were willing to have an online appointment due to COVID-19, and $88.9 \%$ were willing to use teledermatology if COVID-19 had not happened. $32.3 \%$ of patients reported that they had ever missed an appointment not due to COVID-19.

Discussion: Our findings support patient willingness to use teledermatology for treatment and management of their HS.

\section{INTRODUCTION}

Coronavirus disease (COVID-19) impacted healthcare delivery for providers and patients as encounters shifted from inperson to telehealth. Teledermatology has become widespread during this time. Its benefits include reduced wait times and improved access, which support its ongoing use. ${ }^{1,2}$ Teledermatology can be valuable for people with hidradenitis suppurativa (HS) who may require maintenance therapy, as well as flare interventions, for the chronic and acute aspects of this inflammatory skin disease, respectively. ${ }^{3}$ However, we hypothesized people with HS may have concerns or challenges participating in teledermatology due to the location of HS lesions in body areas that are sensitive or difficult to photograph without assistance. The objective of this survey study was to investigate patients' perceived challenges and willingness-to-pay for teledermatology as compared to in-person visits for HS management.

\section{METHODS}

A cross-sectional survey was conducted March 23 - May 1, 2020. The survey was developed and data managed in REDCap, a secure web-based software. ${ }^{4}$ The survey was developed by the authors and pilot tested prior to dissemination. A link to an 
anonymous survey was sent to people with $\mathrm{HS}$ seen at one academic institution and to leaders of three patient networks. In addition, network recruitment was utilized, as participants could share the link with family and friends with HS. Data analysis, including descriptive statistics and Chi square for comparisons, was conducted in May 2020 using SAS 9.4. The study was reviewed and approved by the Penn State Institutional Review Board.

\section{RESULTS}

A total of 149 patients completed the survey (Table 1). The actual response rate is unknown since it cannot be determined what number received the link. Survey results (Table 2) indicate that 140 (94.6\%) patients were willing to have an online appointment due to COVID-19. Additionally, 132 (89.2\%) patients identified they would be willing to use teledermatology if COVID-19 had not happened. Patient-reported concerns with teledermatology were: concern with provider difficulty in assessing HS (74 patients, $50.0 \%$ ), and concern with the security of their photos (64 patients, $43.2 \%$ ) or the security of their information (46 patients, $31.1 \%)$. In total, 38 (26.5\%) respondents missed or cancelled an appointment because of COVID-19. Additionally, 46 $(32.3 \%)$ patients identified that they have ever missed or cancelled an appointment for reasons other than COVID-19. The survey provided possible options for missing appointments other than COVID-19 and the most common responses included: 17 (37.0\%) patients who identified that they were not able to leave work/school/duties at home, as well as 17 (37.0\%) patients who identified that they were feeling really low/depressed and did not feel like attending their appointment.
Table 1. Participant Characteristics $(n=149)$

\begin{tabular}{|c|c|}
\hline Variable & n (\%) \\
\hline \multicolumn{2}{|l|}{ Sex } \\
\hline Male & $12(8.1)$ \\
\hline Female & $136(91.2)$ \\
\hline Decline to Answer & $1(0.7)$ \\
\hline \multicolumn{2}{|l|}{ Age (1 missing) } \\
\hline $18-24$ & $13(8.8)$ \\
\hline $25-34$ & $37(25.0)$ \\
\hline $35-44$ & $52(35.1)$ \\
\hline $45-54$ & $31(21.0)$ \\
\hline $55-64$ & $12(8.1)$ \\
\hline $65+$ & $3(2.0)$ \\
\hline \multicolumn{2}{|l|}{ Ethnicity } \\
\hline Hispanic/Latino & $8(5.3)$ \\
\hline Non-Hispanic/Latino & $137(92.0)$ \\
\hline Decline to Answer & $4(2.7)$ \\
\hline \multicolumn{2}{|l|}{ Race } \\
\hline Asian & $5(3.4)$ \\
\hline Black/AA & $11(7.4)$ \\
\hline American Indian/ Alaska Native & $2(1.3)$ \\
\hline White & $119(79.9)$ \\
\hline Mixed Race & $7(4.7)$ \\
\hline Decline to Answer & $5(3.4)$ \\
\hline
\end{tabular}

\section{DISCUSSION}

Our findings support Shah et al. and their emphasis on the utilization and effectiveness of teledermatology to diagnose, treat, and manage patients with HS. ${ }^{3}$ The results of this study are useful for clinicians so that they can anticipate some of the teledermatology-related concerns or challenges for people with HS. Patient concerns included cost of the teledermatology appointments, security of their photos and information during these visits, and the ability for the provider to adequately assess their HS through these visits. Privacy and accuracy concerns could be addressed by sharing information about the software's security features and demonstrated accuracy of teledermatology ${ }^{5}$, respectively. Regarding cost, telehealth has been covered by insurance during the 
Table 2.

\begin{tabular}{|lcc|}
\hline Have you missed an in-person appointment for your HS or cancel close to the date: & $\mathbf{n}(\%)$ Yes & p value \\
Due to COVID? & $38(25.5)$ & 0.32 \\
Unrelated to COVID? & $46(30.9)$ \\
Most frequent reasons for missed in-person appointment: & $17 / 46(37.0)$ \\
Work, school, duties at home & $17 / 46(37.0)$ \\
Feeling low/depressed & $16 / 46(34.8)$ \\
Too much HS-related pain & $140(94.6)$ & 0.09 \\
Are you willing to have an online appointment with your dermatologist? & $132(89.2)$ \\
Due to COVID? & $140(94.6)$ \\
Unrelated to COVID? & $137(92.6)$ & 0.48 \\
Are you willing to submit photos of your HS to your doctor through the computer? \\
Due to COVID? & $74(50.0)$ \\
Unrelated to COVID? & $64(43.2)$ \\
Most frequent concerns about teledermatology: & $46(31.1)$ \\
Provider difficulty assessing HS & \\
Security of my photos &
\end{tabular}

COVID-19 pandemic and there is pressure to continue this coverage. ${ }^{6}$

There are limitations to this study. The results may not be generalizable due to recall bias or selection bias related to online recruitment. This was a cross-sectional study so cannot determine changes in telehealth use over time or clinical outcomes. Overall, teledermatology was welcomed by our patients and will hopefully be used during this challenging time and thereafter.

IRB Status: The study was reviewed and approved by the Penn State Institutional Review Board

\section{Conflict of Interest Disclosures:}

Kirby: Speaker for AbbVie, Consultant for AbbVie, ChemoCentryx, Incyte, Novartis, UCB

Eichelberger, Silva, Butt, Mosley: No disclosures

Funding: None

\section{Corresponding Author:}

Joslyn S. Kirby, MD, MS, MEd

Associate Professor

Penn State Milton S Hershey Medical Center

500 University $\mathrm{Dr}$

Hershey, PA 17033

Email: Jkirby1@pennstatehealth.psu.edu

\section{References:}

1. Gupta R, Ibraheim MK, Doan HQ. Teledermatology in the wake of COVID-19: Advantages and challenges to continued care in a time of disarray. J Am Acad Dermatol. 2020.

2. Latifi R, Doarn CR. Perspective on COVID-19: Finally, Telemedicine at Center Stage. Telemed J E Health. 2020.

3. Shah M, Naik HB, Alhusayen R. Hidradenitis suppurativa: the importance of virtual outpatient care during COVID-19 pandemic. J Am Acad Dermatol. 2020.

4. Harris PA, Taylor R, Thielke R, Payne J, Gonzalez N, Conde JG. Research electronic data capture (REDCap)--a metadata-driven methodology and workflow process for providing translational research informatics support. J Biomed Inform. 2009;42(2):377381.

5. Lamel S, Chambers CJ, Ratnarathorn M, Armstrong AW. Impact of Live Interactive Teledermatology on Diagnosis, Disease Management, and Clinical Outcomes. Arch Dermatol. 2012;148(1):61-65.

6. Wicklund, E., 2020. Experts Weigh In On Post-COVID19 Telehealth Rules And Policies. [online] mHealthIntelligence. Available at: $<$ https://mhealthintelligence.com/news/experts-weighin-on-post-covid-19-telehealth-rules-and-policies> [Accessed 17 June 2020]. 